\title{
Realization of a Hybrid Face Detecting and Verifying System
}

\author{
Mahmut Dirik \\ Computer Engineering \\ Department, Inonu University, \\ Malatya, Turkey
}

\author{
Davut Hanbay \\ Computer Engineering \\ Department, Inonu University, \\ Malatya, Turkey
}

\author{
A. Fatih Kocamaz \\ Computer Engineering \\ Department, Inonu University, \\ Malatya, Turkey
}

\begin{abstract}
Face recognition is a popular subject in computer vision and objects recognition area because of each person has unique facial features. In this paper, the realization of a hybrid system for face detecting and verifying was presented. Gabor wavelet transform was used to extract facial features of individuals from images. An Artificial neural network was used to classify faces by using obtained features. Phase correlation method was used for face verifying. A MATLAB Graphical user interface was designed by combining these systems for realizing proposed hybrid system, after filtering and scanning methods, the obtained face areas demonstrate within an outline. Phase correlation methods were used to accelerate the searching process. The performance of the proposed system was tested on different image database. It was understood that the proposed method works with high accuracy but is slow when considered as the whole process.
\end{abstract}

\section{General Terms}

Face Recognition, Gabor wavelets, Phase Correlation, Artificial Neural Networks

\section{Keywords}

Face Recognition, Gabor wavelets, Phase Correlation, Artificial Neural Networks

\section{INTRODUCTION}

In image or video processing, finding or identifying objects in an image or video sequence is called object recognition [1]. Biometry can be defined as to identify a person based on the physiological or behavioral characteristics [2]. Different types of biometric technologies are developed by the scientist for identifying or verifying an individual. Face recognition is a popular subject in biometry. One of the methods used in this area is the Fourier transformation. Fourier transform is the most popular tool for analyzing frequency properties of a signal. But the worst side of Fourier is the time information lost, after transformation. So, it is not accurately known where the position of frequency. To solve this problem there is a lot of time-frequency analysis techniques like representing a 1-D signal in time and frequency simultaneously. One of timefrequency analysis technique is wavelet transforms. Time and frequency information can be brought out by the wavelet transform from a signal [3]. Among kinds of wavelet transforms, the Gabor wavelet transform has got magnificent mathematical characteristics and is used frequently in image processing [3]. Gabor wavelets, that uses the properties of spatial frequency and directional selectivity, is an effective object analysis process [4]. Gabor functions are frequently used for feature extraction, especially in texture based image analysis and more practically in face recognition. They are very similar to Morlet wavelets. It is useful to apply several Gabor filters with different orientations and frequencies in order to extract the most significant features in an image [5]. This is used to construct spectral filters for segmentation or detection of certain image texture and periodicity characteristics [5]. Phase correlation was used for image matching in image processing. In principle, phase correlation uses a fast frequency domain to guess the relative connection between two approximate images. Chai, Z., and others suggested a novel image representation method. They named to this method as Histograms of Gabor Ordinal Measures (HOGOM). They developed Gabor Ordinal Measures (GOM) as a new texture descriptor which takes the advantages of Gabor features and Ordinal Measure [6]. Venkatramaphanikumar S. and others presented a new method. They claimed that this new method improves the efficiency of face recognition based on local feature extraction using Gabor Wavelets [4]. Yi J. And Su F. proposed a Gaborbased feature termed Histogram of Gabor Magnitude Patterns (HGMP) which is very simple but effective. HGMP adopts the Bag-of-Words (BoW) image representation framework [7]. Artificial Neural Networks (ANN) were used in many studies for face recognition. One of them is the work of Kolhandai Y. and others [8][9]. In their study, they present an intelligent hybrid feature-based face recognition system that uses the principal component analysis for global features extracting and they used central moment and Eigenvectors and the standard deviation of the eyes, nose, and mouth as local features. Kobayashi, H. Etc. [10] has proposed a method that uses modified linear discriminant analysis (M-LDA) and neural network $(\mathrm{NN})$ for face detection. For feature extraction, The M-LDA was used and the final decision is made by using ANN. Svvides and others [11] offered a new method, in their papers, for incrementing the achievement of robust illumination-tolerant face recognition. They tested this method on test faces which were captured under different illumination, and they showed that this method worked well. The method which they used is a combination of PCA correlation filter. Bhagavatula V. K. and others [12] argued the use of correlation filters for face recognition and demonstrated the advantages of this correlation filters to reduce the computational complexity to increase the accuracy of face recognition. K. lto et al [13] presented a palm print recognition algorithm using phase-based image matching. They achieved highly robust palm print recognition by using of phase components on palm print images. Feng-Xi et al [14] introduced a novel facial feature extraction. They used FERET and AR databases to show result comparison with Eigenfaces linked Discriminant based Coefficient of Variance. In [15], a new frame for face region recognition of selective ensemble face recognition is proposed and also handled SEME, which is substantiation of SEIR based on multiple Eigenspaces. Jun-Zhou et al [16] proposed phase correlation for iris image registration by sub-pixel accuracy. They compared with substantial methods and claimed by 
showing experimental results that the proposed algorithm is encouraging.

This paper is organized as follows: In section II, it is presented the preliminaries of Gabor wavelets, Phase Correlation and Artificial Neural Networks. in Section III, the proposed algorithm for Realization of a hybrid Face Detecting and Verifying System is presented. Section VI contains the conclusion and recommendations.

\section{THE PROPOSED METHODS}

\subsection{Gabor Wavelet Transform}

Gabor wavelet technique used in facial recognition, face tracking, and position estimation, has wonderful features. Gabor wavelets (also called filters) have been used as a robust filter in the literature by using spatial frequency (scaling), localization and directional selection properties. Among various wavelet types, Gabor functions give optimum resolution in the time and frequency domains [17]. Gabor wavelets use the properties of spatial frequency and directional selectivity, an object to be effective for analysis filter. It is a sinusoidal plane wave which is modulated by the kernel function. The spatial domain, a Gabor filter, is a complex structure modulated by a Gaussian function. Gabor wavelet filter can be defined as in Eq. (1) [4] [18].

$\Psi g(x, y)=\frac{f^{2}}{\eta \gamma \pi} \exp \left(\beta^{2} y^{\prime 2}-\alpha^{2} x^{\prime 2}\right) \exp \left(2 \pi f x^{\prime}\right)$

$x^{\prime}=x \cos \theta+y \sin \theta, y^{\prime}=y \cos \theta-x \sin \theta$

where; $\mathrm{f}=$ the center frequency of the sinusoidal plane wave, $\theta=$ the Gaussian clockwise inverse wave, $\alpha=$ the axis parallel to the peak value of the Gaussian wave, $\beta=$ the vertical wave amplitude value of Gauss, $\gamma=\mathrm{f} / \alpha$ and $\eta=\mathrm{f} / \beta$ the ratio between the frequency and the accuracy of the values. As a common feature of the Gabor wavelet transforms used in existing face recognition systems, 40 Gabor wavelets corresponding to 5 scales and 8 orientations were accepted. The real parts of Gabor wavelets are shown in Fig 1.

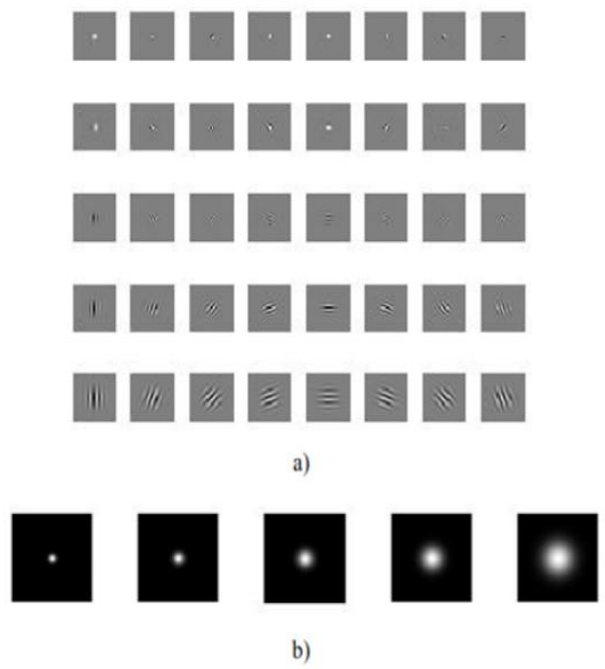

Fig.1. Gabor Wavelets, a) The real part of eight oriented Gabor filters, b) Five Size of different scale Gabor filters

\subsection{Artificial Neural Networks}

In the recent decade, different architectures and Artificial Neural Networks (ANN) models have been used for face recognition. ANN has been used frequently in this field as well as in model recognition and classification. They consist of a large number of interconnected processing units called neurons. These neurons work together to solve the specific problem. The connections between neurons in the network (synaptic weights) are used to store the learned knowledge from the knowledge of learning process, keeping in mind that each neuron used for classification and prediction processes has a threshold or activation function. This is the electronic implementation of the nervous system that processes the working logic of the human brain system. The task of the ANN is to provide an extension system that implements an instance of learning about the event and produces solutions that are generalized to similar events, by setting an exit that can correspond to a given set of data [10] [19]. The determination of face images using Gabor wavelets has been combined with the ANN feature classifier of face motion and face recognition. The correct construction of mathematical models, activation function, input / output signals are important for the functioning of a neuron. The structure of this mathematical equation is given in (3).

$$
y=f\left(\left(\sum_{i=1}^{m} w_{i} x_{i}=w_{1} x_{1}+w_{2} x_{2}+\cdots w_{m} x_{m}\right)+\theta\right)
$$

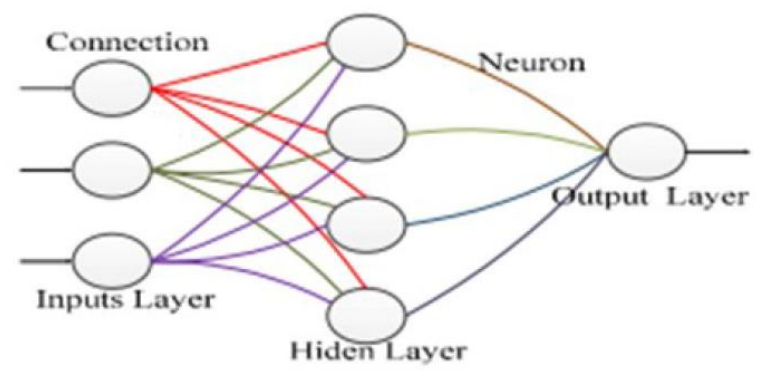

Fig.2. Three-layer ANN architecture.

As shown in Figure (2), a typical ANN has come to an end with an input layer, an output layer, and a hidden layer. There are usually two hidden layers, with the freedom of the number of hidden layers being available. The input-output layers are connected to each other by the intermediate layer. When designing a neural network, it is necessary to pay attention to some issues such as network size, a number of hidden layers, size of input layers and learning parameters according to the probing to be solved.

\subsection{Phase Correlation}

Phase correlation uses the basic properties of the discrete Fourier transform. The method used to detect the similarity of two discrete signal or image correlation. To measure the similarity between two marks is basically, using the properties of Fourier Transform, a phase correlation calculation between two images is shown in Fig. 3 a, b. 


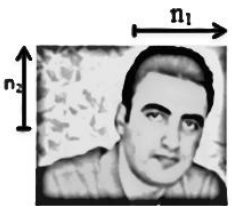

a)

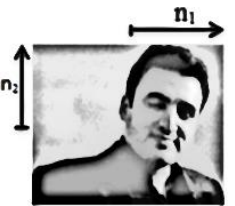

b)
Fig.3. $n_{1} \times n_{2}$ dimensional images a) $f\left(n_{1}, n_{2}\right)$, b) $g\left(n_{1}, n_{2}\right)$

$n_{1}$ and $n_{2}$ expressions, respectively, $n_{1}=-\mathrm{M} 1, \ldots \ldots, \mathrm{M} 1(\mathrm{M} 1>0)$ and $n_{2}=-\mathrm{M} 2, \ldots \ldots, \mathrm{M} 2(\mathrm{M} 2>0)$ given that the range value, in this instance, $\mathrm{N} 1=2 \mathrm{M} 1+1$ and $\mathrm{N} 2=2 \mathrm{M} 2+1$ takes these values. This condition is calculated the same way for $g\left(n_{1}, n_{2}\right)$. Two images for the calculation of the two-dimensional Discrete Fourier Transform (DFT) is formulated as $\mathrm{f}\left(k_{1}, k_{2}\right)$ and $g\left(k_{1}, k_{2}\right)$.

$F\left(k_{1}, k_{2}\right)=\sum_{n_{1=-M_{1}}}^{M_{1}} \sum_{n_{2=-M_{2}}}^{M_{2}} f\left(n_{1}, n_{2}\right) W_{N_{1}}^{-k_{1} n_{1}} W_{N_{2}}^{-k_{2} n_{2}}$

$=A_{F}\left(k_{1}, k_{2}\right) e^{j \theta_{F}{ }^{\left(k_{1}, k_{2}\right.}}$

The expression of this seriatim; $k_{1}=-M_{1}, \ldots M_{1}, k_{2}=$ $-M_{2}, \ldots M_{2}, W_{N_{1}}=e \frac{-j 2 \pi}{N_{1}}$ and $W_{N_{2}}=e \frac{-j 2 \pi}{N_{2}}$ is expressed in the form. $A_{F}\left(k_{1}, k_{2}\right)$ shows amplitude. $\theta_{F}\left(k_{1}, k_{2}\right)$ is show the phase.

$G\left(k_{1}, k_{2}\right)=\sum_{n_{1=-M_{1}}}^{M_{1}} \sum_{n_{2=-M_{2}}}^{M_{2}} g\left(n_{1}, n_{2}\right) W_{N_{1}}^{-k_{1} n_{1}} W_{N_{2}}^{-k_{2} n_{2}}$

$=A_{G}\left(k_{1}, k_{2}\right) e^{j \theta_{G}{ }^{\left(k_{1}, k_{2}\right)}}$

where, $k_{1}=-M_{1}, \ldots M_{1}, \quad k_{2}=-M_{2}, \ldots M_{2}, W_{N_{1}}=e \frac{-j 2 \pi}{N_{1}}$, $W_{N_{2}}=e^{\frac{-j 2 \pi}{N_{2}}}$ and $A_{F}\left(k_{1}, k_{2}\right), \quad \theta_{F}\left(k_{1}, k_{2}\right)$ respectively indicators of amplitude and phase. Cross-Phase Corelation is formulated such that below.

$R_{F G}\left(k_{1}, k_{2}\right)=F\left(k_{1}, k_{2}\right) \overline{G\left(k_{1}, k_{2}\right)}$

$=A_{F}\left(k_{1}, k_{2}\right), A_{F}\left(k_{1}, k_{2}\right) e^{j \theta^{\left(k_{1}, k_{2}\right)}}$

where; $\overline{G\left(k_{1}, k_{2}\right)}$ shows the complex conjugate. $\theta\left(k_{1}, k_{2}\right)$ indicates that the phase difference. $R_{F G}\left(k_{1}, k_{2}\right)$ of inverse discrete Fourier transform on the cross-correlation equation is shown as follows.

$$
\begin{gathered}
r_{f g}\left(n_{1}, n_{2}\right)= \\
\frac{1}{N_{1} N_{2}} \sum_{k_{1=-M_{1}}}^{M_{1}} \sum_{k_{2=-M_{2}}}^{M_{2}} R_{F G}\left(k_{1}, k_{2}\right) W_{N_{1}}^{-k_{1} n_{1}} W_{N_{2}}^{-k_{2} n_{2}}
\end{gathered}
$$

when $\mathrm{f}\left(n_{1}, n_{2}\right)$ and $\mathrm{g}\left(n_{1}, n_{2}\right)$ two similar images, crosscorrelation of these images creates a significant amplitude, if not, the resulting amplitude value is reduced significantly.

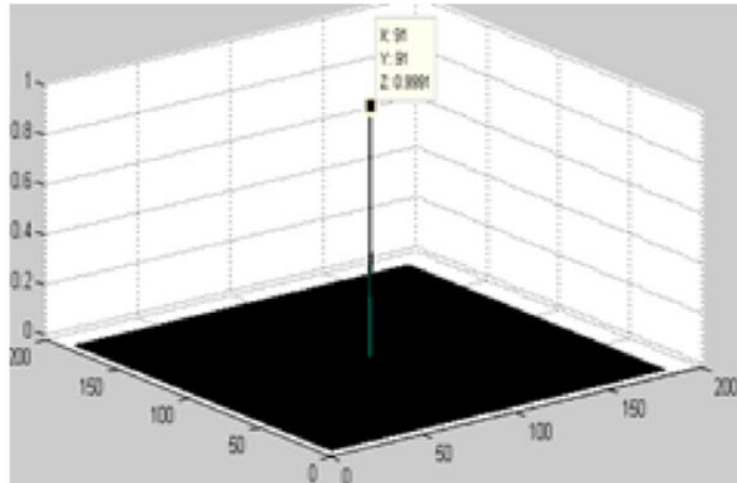

a)

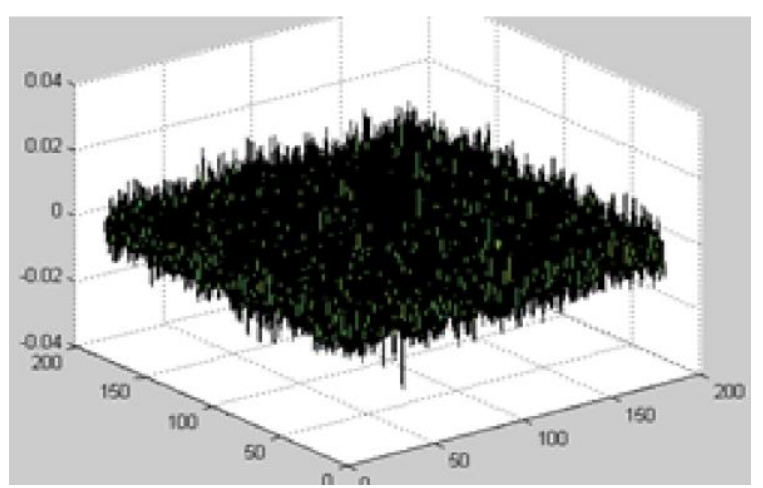

b)

Fig. 5. Cross-correlation, a) Cross-Correlation obtained from the two same images, b) Different and absolute non-amplitude of the cross-correlation obtained from two different images.

\section{EXPERIMENTAL RESULTS}

In this study, a hybrid system for facial recognition and verification was realized. Facial images with complex backgrounds were used to test the performance of the presented face detection and verification method. In the first step of the application, an image database was created. MIT + CMU databases were used. In addition to these, the actual images have been obtained are also were used and tested on randomly selected different images. Gabor wavelet transform was used for feature extraction. These properties were applied to the introduction of artificial neural networks and classification. Phase correlation method is used to illustrate the accuracy of the similarity (see Fig. 10, fig. 11). For face recognition and verification, it is developed a user interface using MATLAB software, as shown in Fig. 6. Here are multiple options to develop different applications. It was understood from the results obtained using this application that this system can be used to achieve the desired result. it is possible to detect faces with different resolutions and background correctly. some operations such as pre-scan have been performed to reduce the application time. 


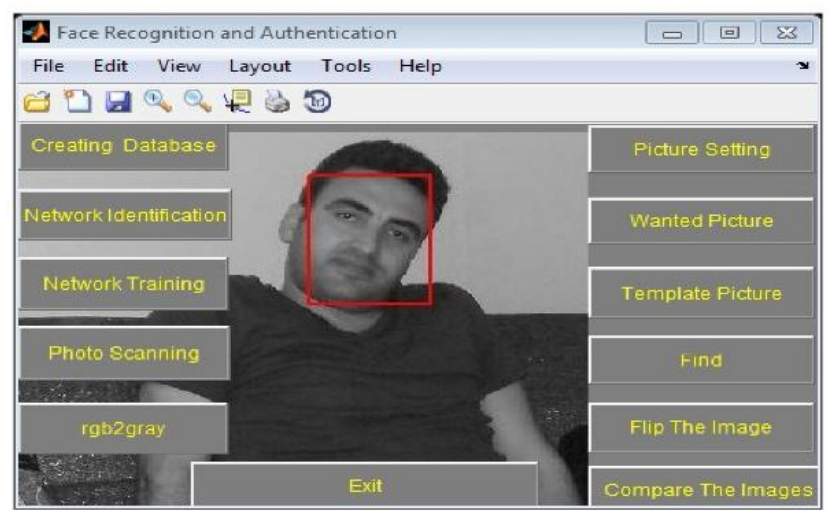

Fig. 6. Graphical User Interface for face detection and verification

\subsection{Image Pre-Screening}

A pre-screening process usually consists of scanning the input image sub-blocks of the picture window. Scan time is calculated by depending on the size of the image and the filter window. The window is shifted over all the pixels in the image. The values standard deviation and variance are calculated according to the bottom of the window. If the threshold is greater than the specified threshold, the middle pixel is selected. If less than the threshold, the center pixel is ignored. The equation developed for this is given in equation (10).

? $\left.\geq \mathrm{UTH}_{\text {可 }}\right) \&\left(\right.$ ? $\left.\leq \mathrm{LTH}_{\text {可 }}\right) \&\left(\delta \geq \mathrm{UTH}_{\delta}\right) \&\left(\delta \leq \mathrm{LTH}_{\delta}\right)$

In Eq. (10), UTH $\mathrm{U}_{\square}$ and $\mathrm{LTH}_{\square}$ are the average value indicates the upper and lower threshold values. $?$ and $\delta$ are indicators of the filter at the bottom of mean and standard deviation. The pre-scan process is being used to detect the searched area for reducing the time of application. This is the advantage of the Pre-scan step. The time required can be reduced by removing the regions near the edges of the image from the center. Because an edge area is unlikely for a face.

\subsection{Face Detection}

The upper and lower threshold values (eq.10) are used as the two-dimensional average value when the face image is obtained. this has been addressed in the image scanning heading. The face area is displayed in a rectangular frame. The determination of the face area in the image, which is a lot of face images, the background color can be influential. there is an effect on the result according to the resemblance of the skin color of the background color. In addition to the detection rate, the speed of detection and the importance of the crosscorrelation method are emphasized in this study.

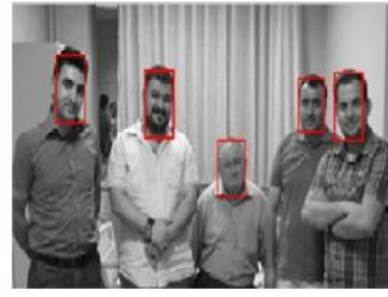

a)

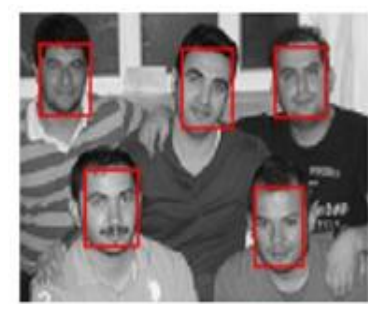

b)
Fig. 7. Face detection test results

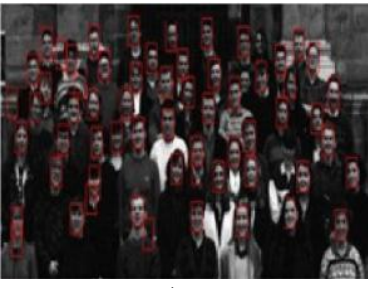

a)

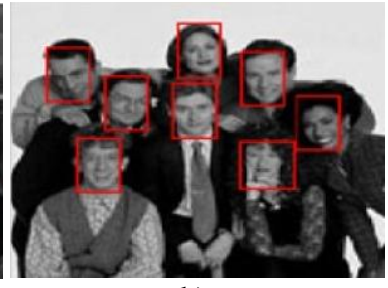

b)
Fig. 8, Sample images from the MIT + CMU database for face detection test results

\subsection{Template Matching}

Phase correlation is a signal correlation method that uses the fundamental properties of the discrete Fourier transform to find the similarity between two discrete signals. The correlation between the two images gives an important idea about the similarity ratio. Cross-correlation methods used to match similarities generate amplitude values according to similarities in images. The peak value of the correlation surfaces of the same surfaces is higher than the peak value of the correlation surfaces of the different surfaces (see fig. 10 and fig 11). The perception of face changes by taking advantage of these properties is performed taking into consideration the greatest value of the correlation frames of consecutive frames. The results of the applications are shown below.

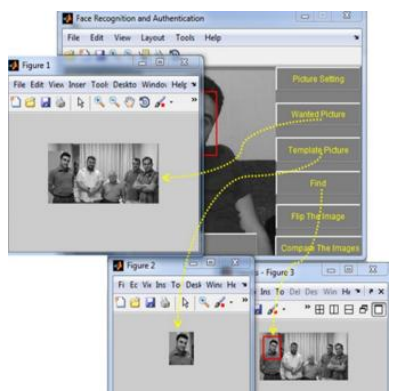

a)

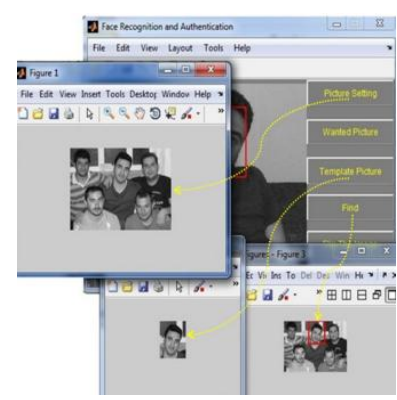

b)
Fig. 9. Template matching applications

\subsection{Image Crosscheck}

The basic properties of the discrete Fourier transform and phase correlation is used for Image matching. By looking the situation two discrete signals or a similar method used to test 
the similarity of the image. The result of the phase correlation is the value of the largest magnitude value $[0,1]$ coming out, and the result is that the two magnitudes comparing the value

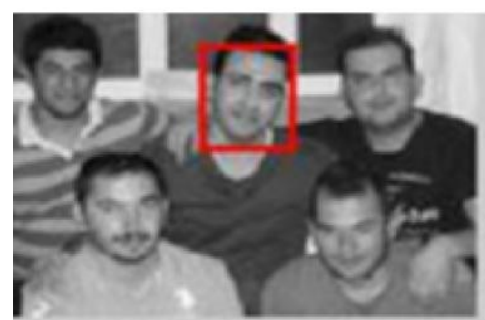

a)

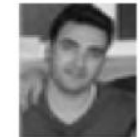

b) closer to 1 are more similar to each other. Detailed information about this topic listed under the heading 2.3 section, "Phase correlation".

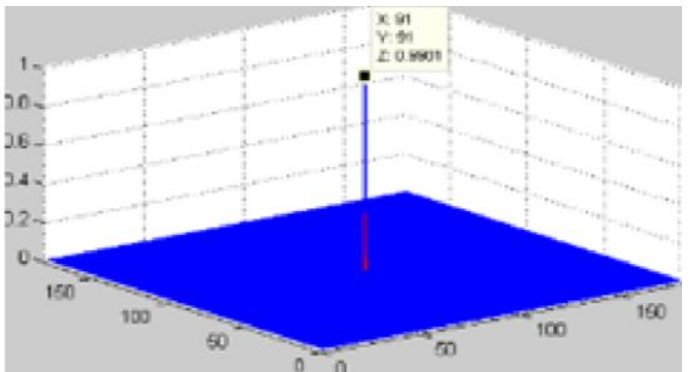

c)

Fig. 10. Phase Correlation, a) The Dialed picture, b) Template Image, c) The Phase correlation obtained for the

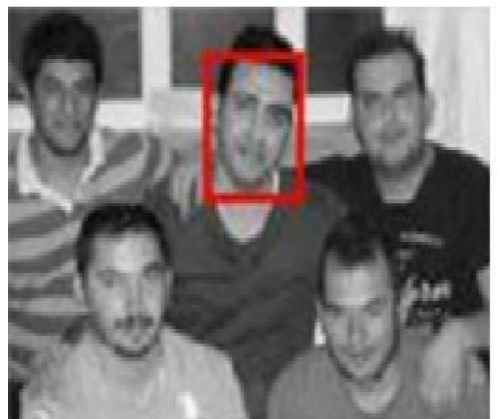

a) same two images.

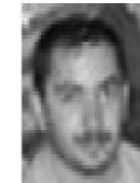

b)

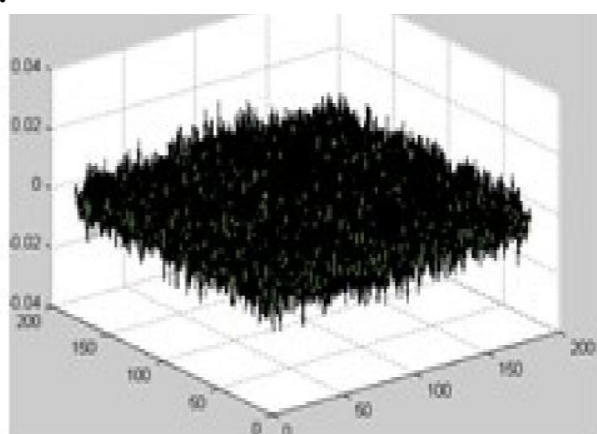

c)

Fig. 11. Phase Correlation, a) The Dialed picture, b) Template Image, c) The Phase correlation obtained for the different two images

\section{CONCLUSION}

In this work, a hybrid system has been developed for face recognition and verification. Gabor wavelets with great features (scaling, localization and directional selection) were used for face detection. After the detection of facial images using Gabor wavelets, artificial neural network model was used to classify facial images. For the convenience of the proposed system, a MATLAB user interface is designed for all these operations. Many results have achieved based on practical experiments. It was understood from the studies made that this system has successfully recognized and detected the human faces in studies performed in different variations. The face images used in the studies performed and the test results of the system were obtained by using various databases. Our proposed method is a combined study that yields alternative and more reliable results than traditional face recognition and finding methods. Automatic face recognition and verification of the person's mental status, emotional depressive characteristics, such as variable characteristics, it is thought that more work on person recognition. it may be necessary to integrate this system with other biometric recognition systems such as voice.

\section{REFERENCES}

[1] W. Ou, X. You, D. Tao, P. Zhang, Y. Tang, and Z. Zhu, "Robust face recognition via occlusion dictionary learning," Pattern Recognit., vol. 47, no. 4, pp. 15591572, Apr. 2014.
[2] K. Ito and T. Aoki, "Phase-based image matching and its application to biometric recognition," in Signal and Information Processing Association Annual Summit and Conference (APSIPA),2013 Asia-Pacific, 2013, pp.1 -7.

[3] Wei-lun Chao, "Gabor wavelet transform and its application." 2011.

[4] V. K. Prasad, "Gabor based face recognition with dynamic time warping," in 2013 Sixth International Conference on Contemporary Computing (IC3), 2013, pp. 349-353.

[5] Á. Serrano, I. Martín de Diego, C. Conde, and E. Cabello, "Analysis of variance of Gabor filter banks parameters for optimal face recognition," Pattern Recognit. Lett., vol. 32, no. 15, pp. 1998-2008, Nov. 2011.

[6] Z. Chai, R. He, Z. Sun, T. Tan, and H. Mendez-Vazquez, "Histograms of Gabor Ordinal Measures for face representation and recognition," in 2012 5th IAPR International Conference on Biometrics(ICB),2012, pp.52 58.

[7] J. Yi and F. Su, "Histogram of Log-Gabor Magnitude Patterns for face recognition," in 2014 IEEE I.

[8] K. Yesu, H. J. Chakravorty, P. Bhuyan, R. Hussain, and K. Bhattacharyya, "Hybrid features based face recognition method using Artificial Neural Network," in 2012 2nd National Conference on Computational Intelligence and Signal Processing (CISP), 2012, pp. 4046. 
[9] K. Yesu, K. Chetia, H. J. Chakravorty, P. Bhuyan, and K. Bhattacharyya, "Innovative feature extraction method for artificial neural network based face recognition," in 2012 3rd National Conference on Emerging

Trends and Applications in Computer Science (NCETACS), 2012, pp. 137-142.

[10] H. Kobayashi and Q. Zhao, "Face Detection Based on LDA and NN," in Japan-China Joint Workshop on Frontier of Computer Science and Technology, 2007. FCST 2007, 2007, pp. 146-154.

[11] M. Sawides, B. V. K. V. Kumar, and P. K. Khosla, "'Corefaces' - robust shift invariant PCA based correlation filter for illumination tolerant face recognition," in Proceedings ofthe 2004 IEEE Computer SocietyConference on Computer Vision and Pattern Recognition, 2004. CVPR 2004, 2004, vol. 2, pp. II834-II-841

Vol.2.

[12] B. V. K. V. Kumar, M. Savvides, and C. Xie, "Correlation Pattern Recognition for Face Recognition," Proc. IEEE, vol. 94, no. 11, pp. 1963-1976, Nov. 2006.

[13] K. lto, T. Aoki, H. Nakajima, K. Kobayashi, and T. Higuchi, "A Palmprint Recognition Algorithm using Phase-Based Image Matching," in 2006 IEEE International Conference on Image Processing, 2006, pp. 2669-2672.

[14] F.-X. Song, D. Zhang, C.-K. Chen, and J.-Y. Yang, "Facial Feature Extraction Method Based on
Coefficients of Variances," J. Comput. Sci. Technol. , vol. 22, no. 4, pp. 626-632, Jul. 2007.

[15] X. Geng and Z.-H. Zhou, "Image Region Selection and Ensemble for Face Recognition," J. Comput. Sci. Technol., vol. 21, no. 1, pp. 116-125, Jan. 2006.

[16] J.-Z. Huang, T.-N. Tan, L. Ma, and Y.-H. Wang, "Phase Correlation Based Iris Image Registration Model," J. Comput. Sci. Technol., vol. 20, no. 3, pp. 419-425, May 2005.

[17] [17] L. Shen and L. Bai, "A review on Gabor wavelets for face recognition," Pattern Anal. Appl., vol. 9, no. 23,pp. 273-292, Oct. 2006.

[18] C. Liu and H. Wechsler, "A Gabor feature classifier for face recognition," in Eighth IEEE International Conference on Computer Vision, 2001. ICCV 2001. Proceedings, 2001, vol. 2, pp. 270-275 vol.2. nternational Conference on Acoustics, Speech and Signal Processing (ICASSP), 2014, pp. 519-523.

[19] H. A. Rowley, S. Baluja, and T. Kanade, "Rotation invariant neural network-based face detection," in 1998 IEEE Computer Society Conference on Computer Vision and Pattern Recognition, 1998. Proceedings, 1998, pp. 38-44.

[20] B. Topcu and H. Erdogan, "Correlation-based patch localization for face recognition," in 2011 IEEE 19th Conference on Signal Processing and Communications Applications (SIU), 2011, pp. 646-649. 